\title{
Functional Significance of Mild Cognitive Impairment in Elderly Patients Without a Dementia Diagnosis
}

\author{
Steven M. Albert, Ph.D., M.Sc., K. Michaels, M.A. \\ M. Padilla, M.A., G. Pelton, M.D. \\ K. Bell, M.D., K. Marder, M.D. \\ Y. Stern, Ph.D., D. P. Devanand, M.D.
}

\begin{abstract}
The authors investigated differences in functional ability among three groups of subjects who were not diagnosed with dementia: normal control $(N C)$ subjects $(\mathrm{n}=35)$; Clinical Dementia Rating Scale (CDRS) score of $O$ (minimal impairment; $\mathrm{n}=26$ ); and CDRS 0.5 (questionable dementia; $\mathrm{n}=42$ ). CDRS $O$ and 0.5 patients reported significantly poorer functioning than NCs in bousebold and other activities, but CDRS O and CDRS 0.5 groups did not differ in self-reported functioning. It is likely that CDRS 0.5 patients overestimated their functional abilities. Correlations between self- and informant reports of functional status were significantly lower in the CDRS 0.5 group than in the CDRS O group, an important finding for clinical management because patients with questionable dementia may actually be more impaired than they admit. Informants' reports or standardized performance-based assessment should be considered in the clinical evaluation of such patients. (Am J Geriatr Psychiatry 1999; 7:213220)
\end{abstract}

$\mathrm{T}$ The functional and neuropsychological status of elderly patients with mild and more severe grades of dementia is well-established in research and specified in staging systems such as the Clinical Dementia Rating Scale (CDRS). ${ }^{1}$ Less is known about the functional status of elderly patients who demonstrate some degree of neuropsychological impairment but do not meet criteria for dementia, a group that has been variously described as (having) "very mild dementia,"2-5 "predementia" (sometimes used interchangeably with "age- associated memory impairment"), ${ }^{6-8}$ "questionable dementia,"1,5,9 "borderzone dementia,"10 or, more generally, "cognitively impaired, not demented."11-16 These elderly patients all demonstrate some degree of cognitive impairment, but fall short of the severity criteria required for a diagnosis of dementia.

Investigation of functional competence in this group of elderly subjects has been limited because most of the studies have been concerned with psychometric differentiation of subjects. However, investigation of the

Received July 20, 1998; revised November 9, 1998; accepted December 7, 1998. From the Gertrude H. Sergievsky Center, College of Physicians and Surgeons, Columbia University, and the Memory Disorders Clinic, Department of Biological Psychiatry, New York State Psychiatric Institute, New York, NY. Address correspondence to Dr. Albert, Gertrude H. Sergievsky Center, Columbia University, College of Physicians and Surgeons, Box 16, 630 W. 168th St., New York, NY 10032; e-mail: sma10@columbia.edu

Copyright (C) 1999 American Association for Geriatric Psychiatry 
functional consequences of mild cognitive impairment in elderly patients without dementia is important for a number of reasons. First, the population of elderly patients with limited cognitive impairment is likely to be large, and information about functional limitation may be valuable for understanding the quality-of-life impact of mild cognitive deficit. Also, it would be valuable to know when neuropsychological deficit, as identified in rather sophisticated psychometric testing, is associated with deficits in independent functioning. In the absence of a functional criterion, raw-score differences in psychometric tests are hard to interpret and may not have clinical significance.

We had the opportunity to investigate these issues in a well-characterized sample of elderly patients who presented for clinical evaluation and met criteria for mild cognitive impairment (MCI), that is, cognitive and functional status between "normal" and "dementia" as per study inclusion/exclusion criteria (see below). These MCI outpatients fell into two categories: "questionable dementia," as operationalized by a CDRS score of $0.5,{ }^{1}$ and "nondementia," with CDRS of 0 , but with evidence of subtle neuropsychological deficits and cognitive complaints. These two groups provided selfreports of functional status, and they also nominated an "informant," usually a spouse or adult child, who also reported on the functional status of the proband. Finally, we recruited a third group of "normal control (NC) subjects." These subjects also underwent all assessments, but did not nominate informants for function ratings.

The presence of the three groups of elderly patients, all of whom were carefully assessed by neurological, neuropsychological, psychiatric, and generalmedical examination, allowed us to investigate the functional significance of states of mild cognitive impairment. Because elderly patients in all three groups were, at most, only mildly impaired, we relied on functional batteries designed to assess high-level functioning. We were particularly interested in the following questions: Is the gradient of cognitive functioning defined by the three groups accompanied by a gradient of functional deficit? We hypothesized that the "mildly impaired but no dementia" group would be intermediate between the other two groups.

A second but related question involves the validity of self-reported functional status. Are self-reports of functional status from elderly patients with questionable dementia as valid as self-reports from elderly pa- tients with minimal cognitive impairment? There are suggestions that elderly patients with executive deficits may be prone to loss of insight as to the extent of functional deficits. ${ }^{17}$ Although we do not have performancebased tests of functional status as an independent criterion, the informant ratings allow us to examine whether correlations between subject and informant ratings in the two groups are similarly correlated.

\section{METHODS}

\section{Subjects}

Subjects for this research were recruited from the Memory Disorders Center at Columbia-Presbyterian Medical Center and the private offices of neurologists who are part of the same center. Informed consent was obtained from the patient and an informant who provided additional data as part of study procedures. The goal was to follow systematically, at 6-month intervals, a broad category of patients who presented with MCI but did not meet DSM-IV criteria for dementia. A group of NC subjects was recruited through three sources: peer nomination (by the MCI patients), NCs who exited other protocols, and local advertising. NCs were followed at annual intervals for comparison purposes, with patients and NC groups matched for age, gender, education, and ethnicity.

For MCI patients, inclusion criteria included age over 40 years and mild cognitive impairment for more than 6 months but less than 10 years. "MCI" was defined in two ways: 1) a diagnosis of "questionable dementia" according to CDRS criteria, ${ }^{1}$ or 2) a diagnosis of "no dementia" (CDRS $=0$ ), with mild cognitive impairment. In making the CDRS ratings, clinicians relied on information from neurologic, psychiatric, and neuropsychological evaluations.

Patients with a score 0 on the CDRS were required to have at least minimal cognitive impairment, defined as scores $\geq 1$ standard deviation (SD) below the norms on one or more of the following neuropsychological tests: recall of two out of three objects at 5 minutes on the modified Mini-Mental State Exam (mMMSE) ${ }^{18}$ delayed recall in the six-trial Selective Reminding Test (SRT), or a Wechsler Adult Intelligence Scale (WAIS) Performance IQ score $>15$ points below the WAIS Verbal IQ score. In two patients with very high premorbid IQ (scores $>140$ ), clear-cut evidence of decline based on 
history led to study inclusion even though neuropsychological criteria were not met. A minimum mMMSE score $>40$ out of 57 (equivalent to $\geq 22$ on the Folstein MMSE) was required for all cases, with the exception that an mMMSE score $>35$ was acceptable in patients with less than 5 years of education. In separate studies, we have found that the mMMSE correlates strongly with the Folstein MMSE and with the Blessed IMC (Information-Memory-Concentration). ${ }^{18}$

Exclusion criteria were a diagnosis of dementia (CDRS score $\geq 1$ ), current schizophrenia, schizoaffective disorder, primary major affective disorder that clearly preceded the onset of cognitive impairment, electroconvulsive therapy (ECT) within the past 6 months, current or recent (past 6 months) alcohol or substance dependence (DSM-IV criteria), clinical or historical evidence of stroke ( $\geq 2 \mathrm{~cm}$. diameter on any MRI slice; periventricular hyperintensities and very small lacunae did not lead to exclusion), cognitive impairment considered to be entirely due to the use of concomitant medications, and the presence of major neurologic illnesses (e.g., Parkinson's disease, amyotrophic lateral sclerosis).

NC subjects were studied annually with the same research procedures as patients with mild cognitive impairment. Potential NCs were screened with the Telephone Interview for Cognitive Status (TICS), an instrument with established reliability and validity that correlates strongly with in-person MMSE interviews. ${ }^{19}$ If no obvious exclusion criterion was met, and the TICS score was $>34$ (out of 41 ), the subject was brought in for testing with the mMMSE and the SRT. For study inclusion as an NC subject, both an mMMSE score $>52$ (out of 57) and SRT delayed-recall score within normal range (score above established norm or no lower than 1 SD below norm) were required. If the subject cleared this cognitive screening, the study neurologist obtained a medical history and examined the subject, focusing on neurologic and psychiatric evaluation. If the subject still met inclusion/exclusion criteria, blood was drawn for a Chem-Screen profile (SMA-20), complete blood count (CBC) with differential, thyroid function tests, vitamin $B_{12}$ and folate levels, and VDRL. Subjects with significant abnormalities were excluded, particularly those that may be etiologic for dementia, for example, low $\mathrm{B}_{12}$ levels, positive VDRL, or untreated hypothyroidism. Subjects with well-controlled disorders (e.g., hypertension, diabetes mellitus, treated hypothyroidism with currently normal thyroid function tests) were eligible to be NCs. During follow-up, none of the NCs have shown marked deterioration on mMMSE $(>15 \%$ decline from baseline) or neuropsychological test scores to date.

\section{Procedures}

For MCI patients, detailed neurological, psychiatric, and neuropsychological evaluations were conducted initially and at annual visits, with shorter evaluations at the intervening 6-month intervals. At initial evaluation, the study physician (neurologist or psychiatrist) obtained a detailed, structured medical history and conducted a physical and neurological examination. Laboratory tests included $\mathrm{CBC}$ with differential, serum electrolytes, liver and renal function tests, thyroid function tests, VDRL, serum $B_{12}$ and folate levels, and either CT or MRI scan of the brain. At annual intervals, the physician completed a brief screen for major depression and psychosis based on DSM-IV criteria and an abbreviated version of the Columbia University Parkinson's Disease Evaluation.

Functional assessments, administered by the research coordinator, included the Instrumental Activities of Daily Living (IADL) scale ${ }^{20}$ and the Pfeffer Functional Activities Scale. ${ }^{21}$ The IADL items assess household management tasks, including the ability to do light shopping, travel to places out of walking distance, do laundry, handle money, take medicines, use the telephone, prepare a meal, and do light housekeeping. Patients and informants were asked if patients had no difficulty, some difficulty, or were completely unable to perform each task. Each was scored dichotomously (No difficulty/Any difficulty), and the sum of tasks with "Any difficulty" (range: 0-8) was calculated for each subject. A similar procedure was used for the Pfeffer scale, which asks about preparing tax records, writing checks, playing games of skill, preparing a balanced meal, using public transportation, shopping alone, and other advanced activities (range: $0-10$ ). Both self-report scales have been validated and have been used extensively in gerontologic research.

At annual intervals, a trained neuropsychology technician administered the following tests: the Wechsler Adult Intelligence Scale-Revised version (WAIS-R scaled scores were used), Wechsler Memory Scale (WMS), Rosen Drawing Test, Controlled Oral Word Association, Selective Reminding Test (SRT; 12-item, 6-trial version; 15-minute delay), selected subtests from the 
Boston Diagnostic Aphasia Evaluation (BDAE, Category Naming and Right-Left Orientation), Boston Naming Test, Benton Visual Retention Test, and Target Finding (shape and letter cancellation tasks). For all subjects, the technician reviewed the neuropsychological evaluation with a senior neuropsychologist (YS) and completed a diagnostic impression and report.

The initial diagnosis was made in a two-step process. First, a clinical team of neurologists, psychiatrists, and neuropsychologists that included the evaluating physician reviewed the clinical evaluation, neuropsychological test results, and laboratory test results in a weekly meeting and reached a consensus diagnosis. Second, two expert raters (DPD and YS) with considerable experience in the clinical evaluation of patients with memory disorders independently reviewed the information from the first consensus diagnosis along with additional available information, for example, the patient's report or behavior during the conduct of the interview, to make research diagnoses. The diagnosis of dementia was based on DSM-IV criteria, and the diagnosis of possible or probable Alzheimer's disease (AD) was based on NINCDS-ADRDA criteria. For the diagnoses of both cognitive impairment and dementia, the most likely etiology (or etiologies) was identified. Any discrepancy between the two expert raters was resolved by consensus, and this consensus diagnosis was used as the primary outcome variable for data analysis.

At initial evaluation, none of the patients were receiving FDA-approved medications for $\mathrm{AD}$ (tetrahydroaminoacridine or donepezil at the time of this study). Only one patient received donezepil (Aricept) in the absence of meeting criteria for dementia during the follow-up period.

\section{Analyses}

Because of our small samples, we first relied on an exploratory, graphical approach. ${ }^{22}$ Box plots showing the distribution of elderly patients on the functional status measures were plotted, showing median scores and the interquartile distribution of scores for each analysis group, as well as the presence of extreme values ("outliers;" subjects whose values were well outside the distribution of group scores). These plots visualize the spread of scores in each group. Nonparametric tests were performed along with traditional parametric tests because of the nature of the functional status outcomes. Because of differences in age and education across di- agnostic groups, these analyses included sociodemographic indicators as covariates.

We assessed correlations between self- and informant reports of proband functional status in the two groups for whom informant reports were available by use of the Pearson correlation coefficient $(r)$. Tests of whether these coefficients were significantly different (that is, whether their difference was significantly different from zero) were performed by use of Fisher's $r$ to- $z$ transformation.

\section{RESULTS}

Forty-two subjects with $\mathrm{CDRS}=0.5,26$ with $\mathrm{CDRS}=0$, and $35 \mathrm{NC}$ volunteers were recruited for this research. The $\mathrm{CDRS}=0.5$ group was significantly older and less well educated than the other two groups. Mean ages for the three groups were 64.2 years (NCs), 61.7 years (CDRS 0), and 71.0 years (CDRS 0.5), respectively; $P<0.001$. Mean years of education for the three groups were 16.2 (NCs), 16.8 (CDRS 0), and 13.5 (CDRS 0.5); $P<0.001$. The groups did not differ in gender composition, with about half female in each group.

Figure 1 shows the distribution of scores on the

FIGURE 1. Modified Mini-Mental State Exam (mMMSE) score, by cognitive status rating (CDRS)

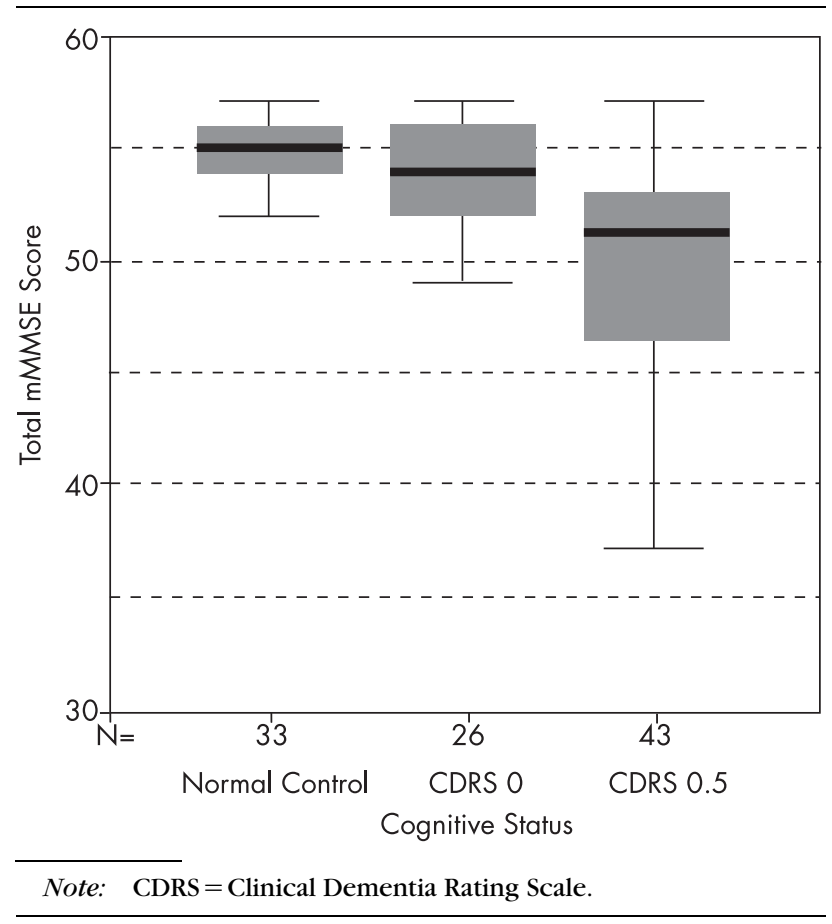


modified Mini-Mental State Exam (mMMSE) test with the box plot procedure (which shows the median line as a bar, plus the frequencies for the two quartiles above and below the median and the full range indicated by boundary lines), and it serves as a check on our recruitment strategies. As the figure shows, mMMSE scores declined across the three groups in the expected pattern. Medians for the three groups were 55 (NCs), 54 (CDRS 0 ), and 51 (CDRS 0.5). Comparison of means (55, 54, and 49 , respectively) showed a significant difference overall ( $P<0.0001$ by analysis of variance), and significant pairwise differences between the CDRS 0.5 group and the other two groups.

Figure 2 and Figure 3 show box plot distributions for the Instrumental Activities of Daily Living (IADL) and Pfeffer Functional Activities scales, respectively, for each cognitive status group. The figures show that CDRS 0 and CDRS 0.5 patients reported poorer functioning on both measures, with higher median impairment and greater dispersion of scores. The KruskalWallis nonparametric test showed significant differences between the groups $(P<0.01$ for IADL; $P<0.001$ for the Pfeffer scale), as did standard analysis of variance ( $P<0.02$ for IADL; $P<0.001$ for the Pfeffer). Significant differences persisted with inclusion of age

FIGURE 2. Self-reported Instrumental Activities of Daily Living (IADL), by cognitive status rating (CDRS)

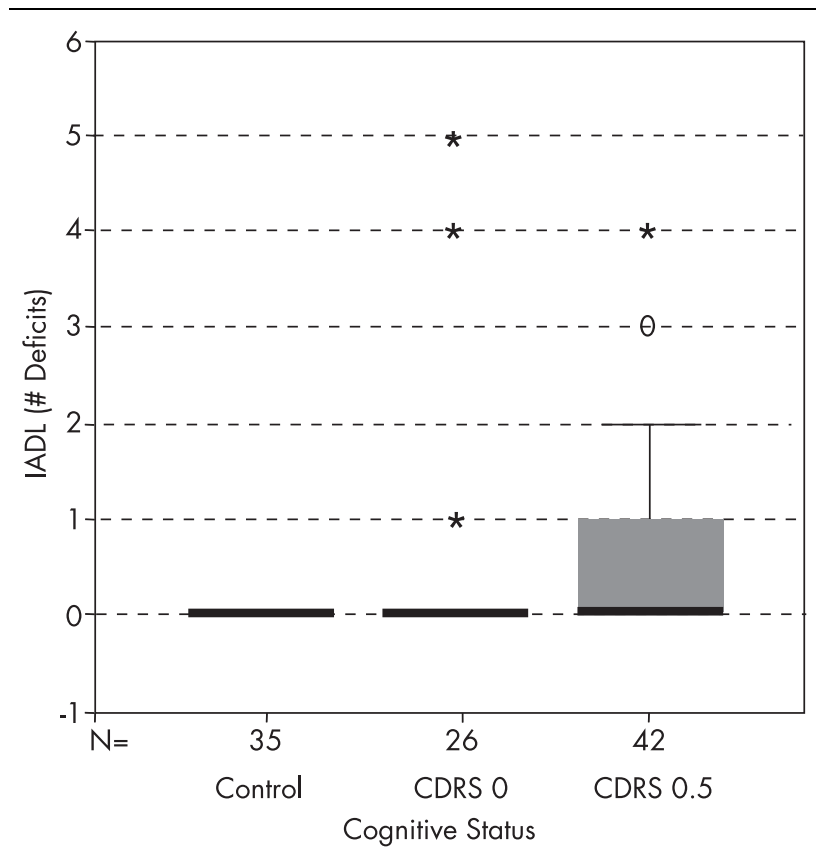

Note: $\quad$ CDRS $=$ Clinical Dementia Rating Scale. Markers indicate single-case outliers. and education as covariates in these models $(P<0.03$ for IADL; $P<0.001$, Pfeffer). In pairwise comparisons, NCs differed from the CDRS 0 and CDRS 0.5 groups, which, however, did not differ from each other.

Informant reports were available for subjects meeting criteria for CDRS 0 and CDRS 0.5. Figure 4 shows box plots for number of IADL deficits as reported by subject informants. Informants reported a greater number of deficits in CDRS 0.5 subjects. Although the median number of IADL deficits in both groups was zero, the interquartile range for CDRS 0.5 subjects extended to one IADL deficit. This difference did not achieve statistical significance in tests of means or in a nonparametric two-samples test, perhaps because of the small sample size. Similar results were found for informants' ratings using the Pfeffer scale.

Absence of difference in self-reported function between the CDRS 0 and CDRS 0.5 groups could reflect differential reporting by patients. That is, CDRS 0.5 patients might have lost insight into the true extent of their deficits. To assess this hypothesis, we plotted the relationship between mMMSE score and self-reported Pfeffer Functional Activities score by cognitive status group. We expected that self-reported deficits would increase with decreasing mMMSE performance. As Fig-

FIGURE 3. Functional Activities Scale score (Pfeffer) and cognitive status rating (CDRS)

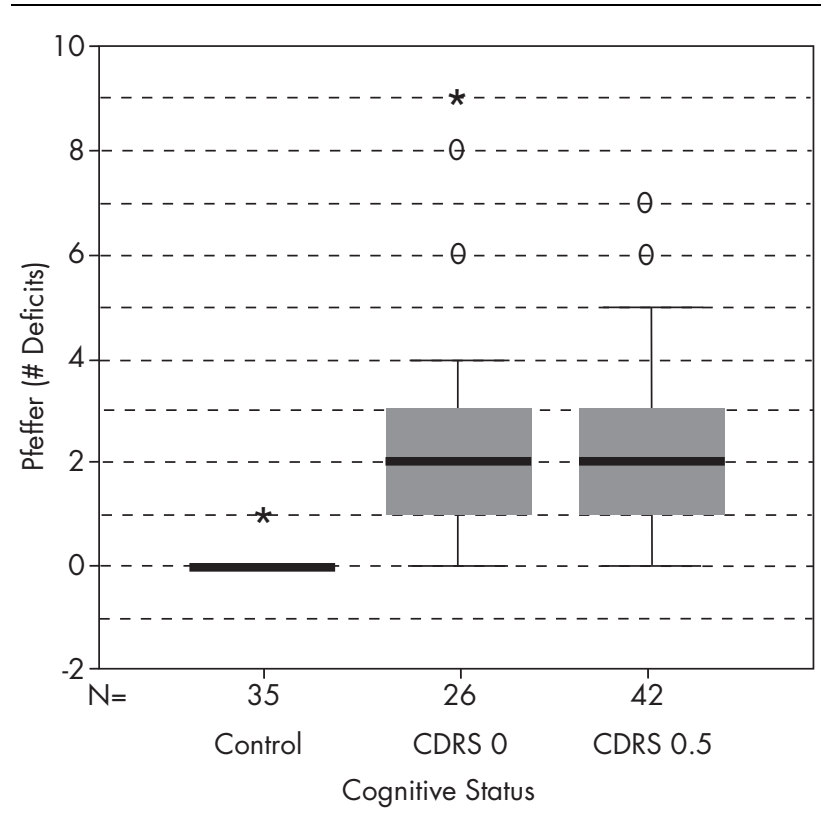

Note: Functional activities score was from the Pfeffer Functional Activities Scale ${ }^{21}$ CDRS $=$ Clinical Dementia Rating Scale. Markers indicate single-case outliers. 
ure 5 shows, this relationship does obtain, but only for the CDRS 0 group. Among CDRS 0.5 patients, self-reported function is not as strongly related to mMMSE status. The figure also shows that NCs were all in the upper range of the MMMSE, with minimal impairment (accordingly, no line could be fit for their score distribution).

The informant reports available for the CDRS 0 and

FIGURE 4. Informant-reported Instrumental Activities of Daily Living (IADL), by cognitive status rating (CDRS)

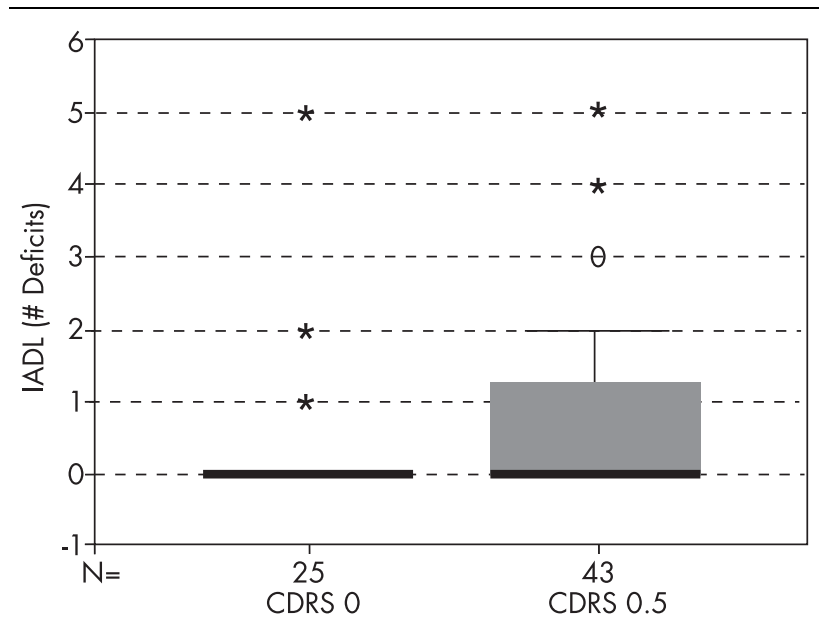

Cognitive Status

Note: $\quad$ CDRS $=$ Clinical Dementia Rating Scale. Markers indicate single-case outliers.

FIGURE 5. Self-reported Functional Activities score (Pfeffer) and mMMSE, by cognitive status rating (CDRS)

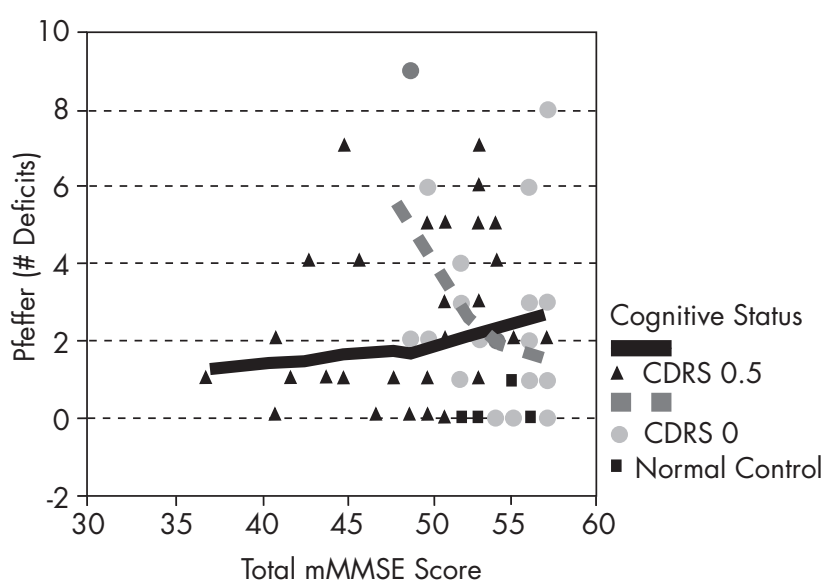

Note: Functional activities score was from the Pfeffer Functional Activities Scale ${ }^{21}$ mMMSE $=$ modified Mini-Mental State Exam; CDRS $=$ Clinical Dementia Rating Scale.
CDRS 0.5 groups allow us to investigate such differential reporting more formally. Correlations between informant and subject Pfeffer ratings were significantly higher among CDRS 0 patients than among CDRS 0.5 patients $(r=0.73$ vs. $r=0.45)$. This difference in correlations was significant $(P<0.05$ by Fisher's $r$-to- $z$ transformation in a one-tailed test). Moreover, inspection of CDRS 0.5 patients with the lowest mMMSE scores showed the greatest disparity between subject and informant reports.

\section{DISCUSSION}

This research has demonstrated differences in selfreported functional ability among subjects without dementia who differ in neuropsychological performance. CDRS 0.5 and CDRS 0 patients reported significantly poorer function than NCs. CDRS 0.5 and CDRS 0 patients did not significantly differ in self- or informantreported function, although trends suggest that CDRS 0.5 patients may be more impaired. Our results also suggest that a subset of CDRS 0.5 patients may overestimate their functional abilities, attenuating true differences between them and CDRS 0 patients. This last finding is important for the clinical management of such patients, because patients with questionable dementia may actually be more impaired than they recognize. Informant reports or standardized performance-based assessment of function should be considered in the clinical evaluation of such patients. Also, this finding supports previous research showing that $\mathrm{AD}$ patients do not reliably report on symptoms they experience. ${ }^{23}$

The Clinical Dementia Rating Scale (CDRS) was developed at the University of Washington, ${ }^{1}$ and its developers report that nearly all patients with a CDRS of 0.5 progress to $\mathrm{AD}^{4,5}$ In our sample of MCI patients, a long follow-up period will be needed to clarify this issue, although in a separate, retrospectively studied sample, we found that a CDRS 0.5 group had heterogeneous outcomes; some progressed to dementia, whereas others did not. ${ }^{24}$ Given the findings regarding discrepancies between patient and informant report in the CDRS 0.5 group, it will be valuable to determine whether subjects with these self-/informant report discrepancies are more likely to develop AD on follow-up. This article presents baseline findings only, but prospective data, currently being collected, should help elucidate this issue. 
Our results suggest that mild cognitive impairment may be associated with greater need for help and restrictions in some daily activities, both of which may lead to earlier use of help services. It is instructive to compare these results with findings reported for population-based surveys. Ganguli and colleagues ${ }^{25}$ reported on the service needs of cognitively impaired elderly patients, defining "cognitive impairment" as scores at or below the 5th percentile on at least one test of memory and one test of another cognitive domain, assessed in an extensive neuropsychological battery. They determined that such cognitive impairment was strongly associated with increased risk of hospital admission over the previous 6 months, as well as increased use of home health services, social services, and prescription medications. In follow-up, our MCI subjects may also show greater use of such services.

A second population-based study, reported by Moritz and colleagues, ${ }^{26}$ showed that poorer scores on a brief cognitive screening tool were associated with increased risk of persistent ADL limitations over 3 years of follow-up. These findings have been supported in a number of other studies. ${ }^{27,28}$ We will determine whether the CDRS 0.5 group has a greater risk of incident ADL deficit than the other two groups on followup.

Some cautions should be considered in interpreting our finding of an association between mild cognitive impairment and functional limitation. This study sample involved elderly patients receiving clinical services, rather than a random sample. Thus, the data presented here cannot be used to establish the prevalence of mild cognitive impairment. Also, the association between mild cognitive impairment and functional limitation may differ in a random sample of elderly patients. The careful characterization of patients and rigorous definition of mild cognitive impairment (MCI) used in this study, however, allow some confidence that MCI has functional consequences that are noticeable to patients and family members.

This research was supported by National Institutes of Health Grant \#MH50038.

\section{References}

1. Hughes CP, Berg L, Daniziger WL, et al: A new clinical scale for the staging of dementia. Br J Psychol 1982; 140:566-572

2. Almkvist $O$, Backman L. Detection and staging of early clinical dementia. Acta Neurol Scand 1993; 88:10-15

3. Rubin EH, Kinscherf DA, Morris JC: Psychopathology in younger vs. older persons with very mild and mild dementia of the Alzheimer type. Am J Psychiatry 1993; 150:639-642

4. Rubin EH, Storandt M, Miller JP, et al: Influence of age on clinical and psychometric assessment of subjects with very mild or mild dementia of the Alzheimer type. Arch Neurol 1993; 50:380-383

5. Morris JC, McKeel DW, Storandt M, et al: Very mild Alzheimer's disease: informant-based clinical, psychometric, and pathologic distinction from normal aging. Neurology 1991; 41:469-478

6. Masur DM, Slivinski M, Lipton DB, et al: Neuropsychological prediction of dementia and absence of dementia in healthy elderly persons. Neurology 1994; 44:1427-1432

7. Jacobs DM, Sano M, Dooneief G, et al: Neuropsychological detection and characterization of preclinical Alzheimer's disease. Neurology 1995; 45:957-962

8. Forstl H, Hentschel F, Sattel H, et al: Age-associated memory impairment and early Alzheimer's disease: only time will tell. Arzneimittel-Forschung 1995; 45:394-397

9. Forsell Y, Fratiglioni L, Grut M, et al: Clinical staging of dementia in a population survey: comparison of DSM-III-R and the Washington University Clinical Dementia Rating Scale. Acta Psychiatr Scand 1992; 86:49-54

10. Gurland BJ: The borderlands of dementia: the influence of sociocultural characteristics on rates of dementia occurring in the senium. Aging 1981; 15:61-84

11. Cooper B, Bickel H, Schaufele M: Demenzerkrankungen und leichtere kognitive Beeintrachtigungen bei alteren Patienten in der artzlichen Allgemeinpraxis. Nervenartz 1992; 63:551-560

12. Ebly EM, Hogan DB, Parhad IM: Cognitive impairment in the non- demented elderly: results from the Canadian Study of Health and Aging. Arch Neurol 1995; 52:612-619

13. Osterweil D, Syndulko K, Martin M: Cognitive function in old and very old residents of a residential facility: relationship to age, education, and dementia. J Am Geriatr Soc 1994; 42:766-773

14. Flicker C, Ferris SH, Reisberg B: A two-year longitudinal study of cognitive function in normal aging and Alzheimer's disease. J Geriatr Psychiatr Neurol 1993; 6:84-96

15. Flicker C, Ferris SH, Reisberg B: Mild cognitive impairment in the elderly: predictors of dementia. Neurology 1991; 41:1006-1009

16. Zaudig M: A new systematic method of measurement and diagnosis of "mild cognitive impairment" and dementia according to ICD-10 and DSM-III-R criteria. Int Psychogeriatr 1992; 4(suppl 2):203-219

17. Grigsby J, Kaye K, Baxter J, et al: Executive cognitive abilities and functional status among community-dwelling older persons in the San Luis Valley Health and Aging Study. J Am Geriatr Soc 1998; 46:590-596

18. Desmond DW, Tatemichi TK, Hanzawa L: The Telephone Interview for Cognitive Status (TICS): reliability and validity in a stroke sample. Int J Geriatr Psychiatry 1994; 9:803-807

19. Mayeux R, Stern Y, Rosen J, et al: Depression, intellectual impairment, and Parkinson's disease. Neurology 1981; 31:645-650

20. Lawton MP, Brody EM: Assessment of old people: self-maintaining and instrumental activities of daily living. Gerontologist 1969; 9:179-186

21. Pfeffer RI, Kurosaki CH, Chance JM, et al: Measurement of functional activities in older adults in the community. J Gerontol 1982; 37:323-329

22. Cleveland WS: Visualizing Data. Murray Hill, NJ, ATT \& Bell Laboratories, 1993

23. Devanand DP, Brockington CD, Moody BJ, et al: Behavioral syn- 
dromes in Alzheimer's disease. Int Psychogeriatr 1992; 4(suppl 2):161-184

24. Devanand DP, Folz M, Gorlyn M, et al: Questionable dementia: course and predictors of outcome. J Am Geriatr Soc 1997; 45:321328

25. Ganguli M, Seaberg E, Belle S, et al: Cognitive impairment and the use of health services in an elderly rural population: The MoVies Project. J Am Geriatr Soc 1993; 41:1065-1070

26. Moritz DJ, Kasl SV, Berkman LF: Cognitive functioning and the incidence of limitations in activities of daily living in an elderly community sample. Am J Epidemiol 1995; 141:41-49

27. Grenier PA, Snowdon DA, Schmitt FA: The loss of independence in activities of daily living: the role of low normal cognitive functioning elderly nuns. Am J Public Health 1996; 86:62-66

28. Gill TM, Richardson ED, Tinetti ME: Evaluating the risk of dependence in activities of daily living among community-living older adults with mild-to-moderate cognitive impairment. J Gerontol Med Sci 1995; 50A:M235-M241 